\title{
STRATEGIES USED IN RHYME-MONITORING
}

\author{
S.M.M. te Riele, S. G. Nooteboom and H. Quené \\ Research Institute for Language and Speech (OTS), \\ Utrecht University, The Netherlands.
}

\begin{abstract}
This study investigates whether subjects use a strategy of word recognition in a rhyme-monitoring task. Results suggest that this is indeed the case. In addition, however, the task introduces an effect of phonological priming of the cue word.
\end{abstract}

\section{INTRODUCTION}

The monitoring task is frequently used in research on auditory word recognition. In such a task, subjects listen for a target unit in a sentence or a list of words. The target unit is usually a phoneme, but it may also be a syllable or even an entire word. One type of target unit that is not very often used is the rhyme. In rhymemonitoring a subject is asked to respond as soon as a word is detected that rhymes with a cue word that was presented in advance (e.g. [2, 4, 5, 6]). A rhyme-monitoring task may be useful when information is required about the processing of the ending of the stimulus word.

When a rhyme-monitoring task is used, it is usually assumed that the subject first recognises words aided by all information present in the context and in the signal. After the target word is recognised, the sound structure of the last part of the cue word is compared to that of the recognised target word. Only if the two words are identical in that respect a response is given.

There are indeed indications that at least lexical information is accessed before a response is given in a rhyme-monitoring task. Faster reaction times are found for target words with the same orthography as the cue words $[2,6]$. In addition, higher level (i.e. syntactic and semantic) information may affect responses. If a semantically related word was presented before the target word, rhyme decisions to these words were faster [2].

However, the results obtained in a rhyme-monitoring task may be affected by phonological priming. It has been shown that one word can prime other words by similarity in phonological structure. Recognition of the target word is facilitated only if the final part of the prime and the target word are phonologically identical [1]. In addition, subjects in word-association experiments sometimes named a word that rhymes with the word to which an associate had to be given [3].Hence, there is an a priori possibility that an effect of phonological priming will occur in the rhyme-monitoring task. After the cue word is presented to the listeners, the recognition thresholds of all rhyming words may be lowered so that these words will be recognised faster. Due to the low recognition threshold, sentence context is not taken into account in these rapid responses.
In this paper a rhyme-monitoring experiment is described. The first question addressed here is whether the subjects in a rhymemonitoring task use a strategy of word recognition. The second question is whether phonological priming plays a role as well.

\section{METHOD}

\subsection{Materials and design}

Half the stimulus material consisted of 10 minimal pairs of twoword phrases. These two-word phrases differed in their underlying representations in that they had a single or a double consonant. An example is the two-word phrase ei slecht 'egg bad' /Ei\#slext/ versus ijs slecht 'ice bad' /عis\#slext/. In the former case, the (single) consonant always belongs to the second word only (/..V\#CV../), whereas in the latter case this post-boundary consonant also occurs in pre-boundary position (/..VCi\#CiV../). According to phonological theory, the contrast between the two interpretations of the minimal pair might be completely neutralised in the phonetic surface form, due to a degemination rule which says that one of two adjacent and identical consonants is deleted. Thus, the realisation /عislext/ may be interpreted as either ei slecht or ijs slecht. All stimulus phrases were noun-adjective combinations, consisting of two monosyllabic and monomorphemic words. The pivotal consonant was always a voiceless fricative.

The second half of the stimulus material consisted of the same nouns as were used in the minimal pair stimuli. They were embedded in two-word phrases that could never be ambiguous in their phonetic surface form, e.g. ei and ijs both not followed by a word starting with an /s/. Examples are ei lekker /عi\#lkkər/ and ijs lekker/Eis\#lekər/ 'egg/ice nice'.

The factors in this experiment are thus: [1] ambiguity: the stimulus may or may not be phonetically ambiguous, and [2] stimulus word type: the stimulus word can be open, i.e. with a single consonant, or closed, i.e. with a double consonant. This results in a total of 40 stimulus words (10 stimulus sets $\mathrm{x} 2$ ambiguity types $\mathrm{x} 2$ stimulus word types).

40 Carrier sentences were created that predicted the stimulus word embedded in that sentence. The sentence context always matched the stimulus word it contained. Thus, contrastive word types (open versus closed) were always embedded in different sentences. Stimulus words occurred at the end of the sentences (but not in last position) so that enough semantic information was available when the stimulus word was presented. 144 Filler sentences were added to the stimulus sentences. Half of these filler sentences contained a 
target word rhyming with the cue word, whereas the other half contained no rhyming target word.

\subsection{Subjects and procedure}

A list of all stimulus and filler sentences in pseudo-random order was read by the second author, who read the sentences at a fast speech rate to ensure proper degeminations. The sentences were resampled at $22.05 \mathrm{kHz}$.

44 Students at Utrecht University, drawn from a list of volunteers, were tested individually in a sound-treated booth. All were native speakers of Dutch with no reported hearing impairment. They were paid hfl 6,50 for their participation. Subjects were divided into two groups. One group of subjects received an open cue word with half of the sentences, whereas the second group of subjects received a closed cue word with these same sentences. This procedure was reversed for the second half of the stimulus sentences. Filler sentences were the same for both groups of subjects.

All stimulus and filler sentences were mixed into pseudo-random order and put in trials. Trials consisted of a cue word, which was followed by a sentence after $1000 \mathrm{~ms}$. Cue words were only presented auditorily to ensure that the sound structure of the mental representation formed by the subjects was correct. Reaction times were measured from rhyme-onset, that is, from the beginning of the vowel in the stimulus word.

The subjects were instructed to push a button as soon as they detected a word that rhymed with the cue word presented in advance. It was explained that a word rhymed with the cue word whenever the vowel and the following consonant (cluster) sounded identical in the cue word and the target word. They were told that the subject who had the fastest reaction times and made the fewest errors would receive a bonus.

\subsection{Expected results}

In addition to reaction times, rhyme-monitoring also provides information about the outcome of the recognition process. If subjects respond after hearing the cue word sprei 'bedspread' and the two-word phrase /عislext/, it can be concluded that they perceived this two-word phrase as ei slecht. If they do not respond they might have perceived this fragment as ijs slecht. The absence of a response in this situation, however, may also reflect lack of concentration by the subject.

The sentence context always matched the stimulus word embedded in that sentence. Hence, both sentence context information and acoustic cues are available to aid recognition of the intended word. Therefore, we decided to classify the outcome of the recognition process into 'errors' and 'correct' responses.

Errors are defined as the recognition of a contextually inappropriate word; they can be divided into false alarms and misses. A false alarm is a response given although the word that rhymes with the cue word is not contextually appropriate and no response was expected. An example is a rhyming response to the cue word sprei and the contextually appropriate two-word phrase ijs slecht. A miss occurs when no response is given although the word that rhymes with the cue word is contextually appropriate and a response is expected. Correct responses, i.e. the case in which a contextually appropriate word was recognised, can be divided into hits and correct rejections. A hit is a correct response given to a contextually appropriate word, whereas a correct rejection occurs when no response was given to a contextually inappropriate word.

When the subjects use a strategy of normal word recognition to perform the rhyme-monitoring task, both sentence context information and acoustic cues are taken into account. Since this context information was always available, even in the phonetically ambiguous stimulus phrases, the contextually most appropriate word is expected to be recognised. Hence, no false alarms should occur.

When phonological priming plays a role, the response may not always be affected by sentence context information. Due to phonological priming, the recognition thresholds of the words rhyming with the cue word are lowered. This might cause the subjects to recognise the word rhyming with the cue word, especially if the sentence context is not very predictive. More importantly, sentence context does not affect responses; hence, false alarms may occur occasionally

As far as the reaction time data are concerned, we expect to find longer reaction times at phonetically ambiguous than at unambigous stimulus words. In the unambiguous stimulus words it will be acoustically clear where the stimulus word ends. In the phonetically ambiguous stimulus phrases, by contrast, both interpretations are acoustically and syntactically possible. Only semantic information can help in deciding which interpretation is correct. Therefore, the subjects will have more difficulty in processing the ambiguous twoword phrases, resulting in longer reaction times at these stimulus words. However, if phonological priming plays a role, the subject may respond as soon as a rhyming word is detected. He then will experience no additional difficulty in dealing with ambiguous twoword phrases and no difference will be observed.

\section{RESULTS}

A log-transformation was performed on the reaction times to obtain normally distributed data. Two analyses of variance, one across subjects and one across stimulus sets were performed on these data. The analyses revealed a significant main effect of ambiguity $\left[\mathrm{F}_{1}(1,9)=16.88, \mathrm{p}<0.01, \mathrm{~F}_{2}(1,43)=34.76, \mathrm{p}<0.01\right.$, $\left.\min \mathrm{F}^{\prime}(1,19)=11.36, \mathrm{p}<0.01\right]$. Table 1 shows that reaction times were longer for the phonetically ambiguous stimulus words than for the unambiguous stimulus words. Thus, subjects seem to have more difficulty in dealing with the ambiguous than with the unambiguous stimulus material, which was expected if a strategy of word recognition is used. Therefore, these results seem to indicate that phonological priming probably did not occur consistently. The main effect of stimulus word type did not reach significance. 


\begin{tabular}{llll}
\hline & Ambiguous & Unambiguous & Total \\
\hline & mean (sd) & mean (sd) & mean (sd) \\
Open Target Word & $579(308)$ & $447(218)$ & $512(274)$ \\
Closed Target Word & $524(218$ & $452(210)$ & $487(254)$ \\
& & & \\
Total & $552(300)$ & $449(214)$ & $499(264)$ \\
\hline
\end{tabular}

Table 1 :Mean reaction times and standard deviations measured from rhyme-onset in ms. Phonetically ambigous and unambiguous stimulus words by open and closed words. Observations in each cell are based on 220 observations.

In Figures 1 and 2 data are presented on the errors and correct responses made by the subjects. Figure 1 shows that false alarms occurred all four conditions, despite the presence of contextual and acoustic cues to the intended word. In three conditions a sequence of sounds that constitutes a rhyming word can always be detected, viz. in condition 1 (ei slecht), 2 (ijs slecht) and 4 (ijs). In these conditions both the word $e i$ and $i j s$ can be found in the string of phonemes. In condition 3 it is never possible to detect a closed word that rhymes with the cue word: In ei lekker /Eilkkər/ the sequence /عis/ is not present. The fact that in this condition $3 \%$ of the responses were false alarms may indicate lack of concentration by the subjects.

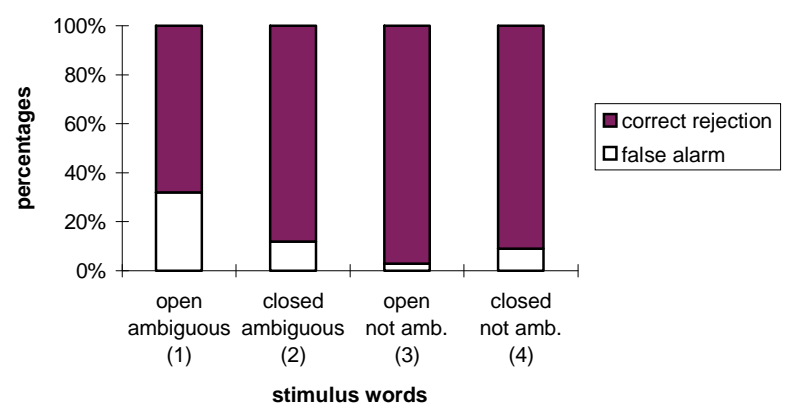

Figure 1:Percentages of false alarms and correct rejections over all items in which the word rhyming with the cue word was contextually inappropriate and no response was expected. Percentages in each bar are based on 220 observations.

More false alarms were obtained in conditions 1,2 and 4 which contain both an open and a closed word. In conditions 2 and 4 about $10 \%$ false alarms were obtained, whereas in condition $332 \%$ of the responses were false alarms. If sentence context were used, no false alarms would be expected. The occurrence of false alarms thus indicates that sentence context was not always taken into account in deciding whether or not to respond.

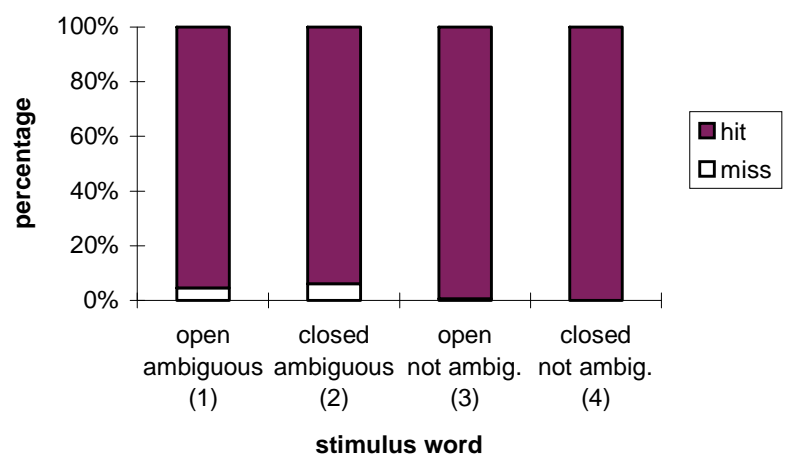

Figure 2:Percentages of misses and hits over all items in which a word rhyming with the cue word was contextually appropriate and a response was expected. Percentages in each bar are based on 220 observations.

Figure 2 shows that misses were hardly ever made, and that they were mostly made in the conditions with ambiguous stimulus words, viz $4.5 \%$ and $6 \%$ in the open (1) and closed words (2), respectively. This can either be caused by lack of concentration by the subjects, or be a result of the subjects sometimes recognising the word that was not intended in that sentence, despite the presence of contextual and acoustic cues to the intended word.

\section{DISCUSSION}

Assuming a strategy of word recognition, we expect longer reaction times at ambiguous than at unambiguous stimulus words. In the ambiguous stimulus material only contextual information can help in segmenting the two-word phrases correctly, whereas in the unambiguous stimulus words, acoustic information is also present. Under the same assumptions we also expect very few or no false alarms, since the available contextual and acoustic cues could be used to recognise the intended word. This is even true in the ambiguous two-word phrases. Thus, the subjects always should be able to recognise the intended word, and no false alarms should occur.

The results show that reaction times were significantly longer at ambiguous than at unambiguous stimulus words. This corresponds to what was expected according to a word recognition strategy. It indicates that subjects indeed had more difficulty in dealing with the ambiguous stimulus material. However, instead of finding very few or no false alarms, about $10 \%$ false alarms were obtained in the closed stimulus words. In the open, ambiguous stimulus word condition, in $32 \%$ of the cases the subjects neglected sentence context information and acoustic cues, and indicated to have detected a closed rhyming word. Thus, false alarms were made more frequently than was expected according to a word recognition strategy. The word recognition strategy is, therefore, not used consistently in a rhyme-monitoring experiment.

A different process seems to play a role as well. The number of false alarms indicates that the subjects sometimes did not take sentence context into account. They recognised a word that was contextually inappropriate but rhymed with the cue word. This is 
expected if phonological priming plays a role. Due to phonological similarity of the cue and the target word, the target word may be primed phonologically. This could be modeled by lowering the threshold of all rhyming words in the mental lexicon, thus facilitating their recognition. This effect may be enlarged by the task: The subjects were explicitly instructed to monitor for rhyming words, so their attention may be focussed even more on these words. Contextual and acoustic cues may have been too weak in some cases to activate the intended word sufficiently. Thus, in these cases the primed rhyming word is detected instead of the intended word, which results in a false alarm. Sentence context effects are then not reflected in the response.

Thus, it seems as if the subjects in a rhyme-monitoring task generally use a strategy of word recognition, using acoustic cues and contextual information. However, at the same time, phonological priming plays a role as well. This results in the lowering of the thresholds for all rhyming words. If sentence context and acoustic cues are strong enough, the contextually most appropriate word is recognised, and no false alarm is made. However, if the sentence context is too weak, the effect of phonological priming may not be overruled, resulting in a false alarm.

Considering the above, the occurrence of false alarms in rhymemonitoring may be an artifact of the task: Since the cue word and the target word are phonologically similar, and since the task requires the subjects to focus on rhyming words, the thresholds of all rhyming words will be lowered. This will facilitate the recognition of a rhyming word, especially if sentence context is not very predictive. Thus, in a certain percentage of the cases a false alarm occurs. The occurrence of false alarms in a rhymemonitoring task may pose a problem, since it will obscure the information about the outcome of the recognition process. However, since the number of false alarms is expected to vary as a function of sentence context predictability, the false alarm rate may also be considered as a dependent variable in itself.

Finally, the results show a response bias to closed words. More false alarms were obtained in the open, ambiguous stimulus word condition than in the closed stimulus word conditions. The response bias may be a consequence of closed words being more frequent or of closed words containing more acoustic information. Under both explanations the subject may be more certain of his response for closed words.

\section{CONCLUSION}

From the present experiment it can be concluded that subjects in a rhyme-monitoring task generally use a strategy of normal word recognition, using all the information at their disposal, e.g. not only acoustic information, but contextual information as well. Therefore, the rhyme-monitoring task seems to be useful in auditory word recognition research where context is a factor.

However, due to characteristics of the task, phonological priming also plays a role. This causes the thresholds of all rhyming words to be lowered, thus facilitating the recognition of these words. Sentence context information is sometimes not sufficient to overrule the effect of the threshold lowering, resulting in a false alarm. The effect of acoustic and contextual information is then obscured.

Finally, it can be concluded that the false alarm rate functions as a dependent variable in itself, since the number of false alarms probably depends on sentence context predictability.

\section{REFERENCES}

1. Cutler, A. and Chen, H.-C. "Phonological similarity effects in Cantonese word recognition", Proceedings of the XIIIth International Congress of Phonetic Sciences, vol. 1, 106-109, 1995.

2. Donnenwerth-Nolan, S., Tanenhaus, M.K. and Seidenberg, M.S. "Multiple code activation in word recognition: Evidence from rhyme monitoring", Journal of Experimental Psychology: Human Learning and Memory, 7, 170-180, 1981.

3. Groot, A.M.B. de Mondelinge woordassociatienormen. Lisse: Swets \& Zeitlinger, 1980.

4. Marslen-Wilson, W.D. and Tyler, L.K. "On the temporalstructure of spoken language understanding", Cognition, 8, 1-70, 1980.

5. McQueen, J.M. "Rhyme decisions to spoken words and nonwords", Memory and Cognition, 21, 210-222, 1993.

6. Seidenberg, M.S. and Tanenhaus, M.K. "Orthographic effects on rhyme-monitoring", Journal of Experimental Psychology: Human Learning and Memory, 5, 546-554, 1979. 